underneath, letting through the light and possibly the air which otherwise would be excluded.

Many plants related to the Arums produce large leaves. A Central American species known as Dracontinum gigas has been known to develop leaves which were no less than fourteen feet long. It is said that the whole plant is a most remarkable spectacle from the fact that the stem of this colossal lea is beautifully mottled with purple and yellow, and has been compared "to a huge snake standing erect at the bidding of an Eastern charmer." But eve here we do not reach the limit of what is possible in the way of giant foliage. Some of the achievements of the Sago palms in this direction throw everything else into the shade. Not so long ago on which, it may be observed, was in captivity, startled its owner by sending out a leaf which was estimated to be more than forty feet in measurement. As matter of fact it is likely that this particular specimen would have been even longer than this had it been possible to allow it a freer growth than it could get under glass. Of course, in the open under ver favorable conditions even this would be exceeded though, as it may be imagined, the chances that such a great leaf would come to grief are very great.

As a general rule the water plants can not be said to be the possessors of very large leaves, but to this there is one very striking exception. Most people are now more or less familiar with the giant water lily (Victoria regia), specimens of which are not uncom monly to be seen in botanical gardens. Perhaps few are aware that this plant is an annual, the whole of its enormous growth being made in a few months. On account of the fact that the edge of the leaf of this water lily is turned up, its supporting power is this water lily is turned up, its supporting power is
very considerable. Very fine examples have been known to bear a man sitting on a chair, although it is only fair to say that the average leaf would not support such a weight.

In reviewing the question of big leaves it would be out of place not to refer to what man has done to increase the size of the foliage of plants for his own ends. This is well instanced in the case of the cabbage, a plant for whose ancestry we must look to a most insignificant species with small leaves. It is perhaps one of the greatest triumphs of the horticultural world that out of this useless plant (Brassica oleracea) has been create such a vast number of varieties of such great value to the whole race of mankind. The cabbage only produces its large leaves because it has been made to so, and it will soon revert to something like its natural state if left un surrounded by all the stimuli of artificial culture for any time.

\title{
THE FORM AND DIMENSIONS OF THE SUN."
}

\author{
POS S I B LE C H A N GES.
}

\section{B Y PROF. F. R. MOULTON, U N I ERS I TY OF CHICAGO.}

THi: shape of the sun and the possibility of changes in its dimensions have been matters of keen interest to astronomers for about a century. Among those who have contribute to the discussion of these question we may mention Von Lindenau (1809), Bianchi (1831), Secchi (1871), Auwers (1873 and 1895), Newcomb an Holden (1874), Ambronn (1905), and Poor (1908). In the last of Poor's papers there are full references and complete abstracts of all the most important discussions of the subject. It is sufficient to state here that up to Auwers's work in 1885 the observations were obtained with meridian instruments, that the later observations of Auwers and those of Ambronn wer servations of Auwers and those of Ambronn were
made with the heliometer, and that Poor's work, aside from a rediscussion of the heliometer measures of Auwers and Ambronn, was based upon twenty-two Rutherford plates taken in the years 1870-72, nine Northfield plates taken in 1893-94, and six Yerkes Observatory plates taken in 1907. In using these photographic plates, Poor limited his discussion to the question of the difference in the equatorial and polar question of

The results obtained in the various investigations have been most discordant. Von Lindenau reached the conclusion that there are periodic variations in the sun's diameter; from the same observation Auwers came to the opposite conclusion; Secchi found the diameter varying inversely with the number of sunspots; Auwers, from the same data, tound no variations; Newcomb and Holden reached the result that there is no long-term variation; in his first discussion of the heliometer observations Auwers found that the diameter varies directly as the number of sun-spots, and in a second discussion that the apparent variation was due to varying personal equations; and Ambronn was convinced that during the interval of thirteen years covered by his observations there was no periodic or secular change in the diameter of the sun exceeding $0.1 \mathrm{sec}$.

The conclusions respecting the shape of the sun were equally varied, the polar diameter being found a little greater than the equatorial about as often as the opposite. Poor infers from all the evidence that "the exact shape of the sun is not known with certainty"; and that the difference in the polar and equatorial radii is probably not more than $0.25 \mathrm{sec}$. He states that the heliometer observations show a fluctuation in the size of the sun of probably not over $0.10 \mathrm{sec}$. Am bronn thinks there is no observational evidence of changes in its dimensions.

Under the hypothesis that the sun is rotating with out oscillations, we find that it must be nearly an oblate spheroid, and that the difference between its equatorial and polar diameters must be less than $0.07 \mathrm{sec}$ as seen from the earth.

It is well known that such a fluid mass as the sun may undergo oscillations whose amplitude and character depend upon the nature of the disturbances pro ducing them, and whose periods depend upon the character of the oscillations, and upon its mass, density, and elasticity. Whatever the nature of the oscillaand elasticity. Whatever the nature of the oscilla-
tions, the question of the surface of the body can be represented as the sum of a converging series of spherical harmonics whose coefficients are periodic func tions of the time. For simplicity, it is assumed that the mass always has an axis of symmetry so that its surface can be defined by zonal harmonics. It is also assumed that at every instant the mass is homogeneous throughout. With these restrictions, the surface volume, moment of inertia, and self-potential of this mass of general variable form are computed.

The first practical question is whether a dilatational oscillation will not change the rate of rotation so that the change of period of rotation can be observed even though the change in diameter is beyond direct observation. The answer is in the negative, for a shrinkage of diameter of 1.0 sec., as seen from the earth, would decrease the period of rotation by only $38 \mathrm{~min}$ utes.

The second question is regarding the change in temperature produced by a dilatational oscillation. An oscillation of this type such that the whole change in the sun's diameter as seen from the earth is only 0.1 sec. is, assuming the specific heat of the sun as unity 1,400 leg. C.

It is found then, assuming Stefan's law of radiation, and that the absolute temperature of the sun at mean radius is 6,000 deg., that a variation of temperature 700 deg. each side of this mean would result in a variation from 61 per cent to 156 per cent of that at mean tion from 61 per cent to 156 per cent of that at mean
radius. That is, the radiation at maximum would be 2.56 times that at minimum, or a variation of little 2.56 times that at minimum, or a variation of little
more than a star magnitude. Consequently, if the sun were oscillating in this way the changes in its rate of radiation would be observed long before those in it diameter. An extreme oscillation of $0.01 \mathrm{sec}$. in the sun's diameter as seen from the earth would account for the variations of 10 per cent in the solar radiation observed by Langley and Abbot.

The question of oscillations defined by zonal har monics of the second order is considered. The potential energy is a minimum when the coefficient of this harmonic passes through zero, that is, at the instant when the body is spherical; and has two equal maxima when the body is most prolate and most oblate. But when the potential energy is a minimum the kinetic energy of the oscillation is a maximum. Consequently the range of variation of temperature cannot be exactly compute without knowing the motion of each element of mass at the critical times. Since this is an unsolve problem, a rough computation was made to determine an upper limit to the kinetic energy at the time the mass is spherical, and therefore a lower limit to the range of variation of temperature. It was found that a variation of this type such that the equatorial diameter changes by $1 / 70$ its value would cause a variation of temperature of $1,600 \mathrm{deg}$. C. At its greatest oblateness it would be relatively flattened onefourth as much as Saturn is now. The radiation at maximum would be 2.6 times that at minimum, or more than a star magnitude greater. An oscillation of this type producing a change of 10 per cent in the solar radiation would cause a variation in the sun's equatorial diameter, as seen from the earth, of $7.4 \mathrm{sec}$ The period of these oscillations is of interest. $\mathrm{Kel}$ vin's formula shows that if the sun were a perfect incompressible fluid the period of the oscillations depending upon the harmonics of the second order would be 3 hours and 8 minutes. The period is independent of the mass and varies inversely as the square root of the density. The work of Lamb shows that if the mass had the viscosity of water the amplitude of the oscillation would be reduced to 37 per cent of its original value in $22 \times 10^{14}$ years. The former of these two results is almost certainly of the right order of magnitude for the actual sun; the latter is probably much too great.

The results obtained by me suggest the idea that certain classes of variable stars may owe thefr variability, at least artially, to oscillations. Reference is made particularly to stars whose light changes con- tinually, such as those of which $\delta$ Cephei is the type, and those of the $\beta$ Lyræ type. The spectroscope proves that in the $\delta$ Cephei stars the light-variations are not eclipse phenomena, and in case of $\beta$ Lyræ the phenomena are so complicated as to suggest that eclipses are not the sole cause of the variability. If oscillations of sufficient magnitude are admitte they would explain rather satisfactorily most of the phenomena of these classes of stars. For a star of the mass and density of the sun an oscillation deflned by the second-order harmonic amounting to $1 / 50$ of its radius would acount for all the light changes in these classes of stars. However, the periods of variability demand very low densities, yet greater than one-third of those required if we attempt to explain the light variations on the eclipse theory. For a period of 5 days the density is $10^{-3}$ on the water standard.

The question at once arises as to the cause of the possible oscillations. In the case of a binary system having an eccentric orbit, the periodically changing disturbance of one star by the other is an efficient cause. A single star would be set vibrating by a collision with another body even of planetary mass, and by passing near another star. The fact that the sun now has a remarkable equatorial acceleration, as well as an unexplained 11-year cycle, tends to make us openminded regar

\section{THE SCHOOL ROOM DUST PROBLEM.}

Ove of the most unpleasant things with which teachers in public schools have to contend is the method of sweeping and cleaning the school rooms. In most cases the rooms are dry swept, which means that the dust and dirt is made the vehicle for the distribution of many pathogenic germs. This dust evil is one of the greatest problems of sanitation left for public school authorities to solve. Much attention has been given, as it should be, to the proper heating and ventilating of our school buildings. The food and water supply have received much attention. It is seen that the plumbing is properly set and in many schools fire escapes have been provided. These things are all good, and it is well that they are being looked after with much care and intelligence. This dust problem, in many respects the most vital of them all, has receive the least attention. Dry sweeping, instead of removing the dirt, divides it into finer particles which float in the air and are finally settled on everything in the room to be stirred up the next ay by the pupils and taken into their lungs. The wonder is that we not have more pupils infected with one form or another of tuberculosis.

The eradicating of the dust nuisance lies in the installation of a vacuum cleaning apparatus in every school, and for that matter, in every public building. In new buildings the extra cost would not be very much, as the exhaust pipes would be placed in walls during construction, all leading to the exhauster in the heating room. The extra expense would soon be met by the saving in cleaning materials and janitor service. The crayon dust nuisance may also be overcome by using vacuum blackboard erasers, which are the very latest things in school building equipment.

Mr. Robert L. Cooley, principal of the Sixteenth Dis trict School No. 1 of Milwaukee, Wis., has had installed in his building a vacuum cleaning apparatus, and his testimony is nothing but praise for its successful work ing in his school. He says the system has completely solved the dust nuisance with him.-School Science and Mathematics. 